\title{
Diseño de nuevos planes de estudios en el contexto del Espacio Europeo de Educación Superior (II). Perfil profesional del graduado en Medicina
}

\author{
J. Carreras
}

En España, para ejercer la profesión médica como médico especialista y para ocupar un puesto de trabajo, público o privado, con dicha denominación se requiere poseer la titulación de la correspondiente especialidad médica. Además, desde el año 1995, por directiva de la Unión Europea, para el ejercicio de las funciones de médico generalista en el marco del régimen público de la Seguridad Social se requiere una formación específica, que proporciona la especialidad en Medicina Familiar y Comunitaria. De acuerdo con ello, el objetivo de la actual licenciatura en Medicina es proporcionar la denominada 'formación médica de base'; aquella formación que debe adquirir todo graduado en medicina con independencia de su orientación futura. Sin embargo, desde la Ley de Ordenación Universitaria del año 1943, la legislación española ha determinado que el título de Licenciado en Medicina habilita para el ejercicio de la medicina, en cualquier ámbito o especialidad, con tal de que no se ejerza con el carácter de especialista. Esta inconsistencia, que según parece va a perpetuarse en la futura titulación de Médico adaptada a los requerimientos del Espacio Europeo de Educación Superior, supone una gran dificultad para el diseño de un plan de estudios coherente basado en competencias establecidas de acuerdo con el perfil profesional del graduado. No son las mismas las competencias que requiere 'todo graduado en medicina', que las competencias que requiere un graduado legalmente habilitado para 'ejercer en cualquier ámbito o especialidad de la medicina', auque sea 'sin el carácter de médico especialista'.

Palabras clave. Espacio Europeo de Educación Superior. Perfil profesional. Planes de estudios de Medicina.
The design of new medical curricula in the context of the European Higher Education Area (II). Professional profile of the medical doctor

In Spain, to practice as a physician in any medical speciality, it is necessary to possess the corresponding specific academic title. And since 1995, following the European Union directives, to exert as medical generalist in the public Social Security System it is required a specific academic formation that is provided by the speciality of Familiar and Community Medicine. Accordingly, the aim of the Spanish M.D. title ('Licenciado en Medicina') is to provide the denominated 'basic medical formation': the formation needed by any physician with independence of his future specialization. However, the Law of Ordination of the Spanish University of 1943 established that the university M.D. title qualifies for the practice in any area of Medicine, if the qualification of medical specialist is not used. Such a contradiction makes very difficult the design of a medical curriculum based on the competences needed by the graduated in Medicine, as it is recommended by principles of the European Higher Education Area.

Key words. European Higher Education Area. Medical curricula. Professional profile.

\section{Introducción}

Es objeto de debate si las competencias que deben proporcionar las titulaciones universitarias han de establecerse siempre a partir de los perfiles profesionales [1]. Pero, si bien ello puede ser
Departamento de Ciencias Fisiológicas I. Facultad de Medicina. Universitat de Barcelona. Barcelona, España.

Correspondencia Dr. Josep Carreras Barnés. Departamento de Ciencias Fisiológicas I. Facultad de Medicina. Universitat de Barcelona. Casanova, 143. E-08036 Barcelona.

Fax +34934035882

E-mail jcarreras@ub.edu 
discutido en el caso de las titulaciones orientadas fundamentalmente a la consecución de objetivos académicos, dirigidos a la formación para la reflexión y el desarrollo del pensamiento, o para la investigación y la génesis del conocimiento, se halla fuera de duda en el caso de aquellas titulaciones que habilitan legalmente para el ejercicio de profesiones tituladas con perfiles claramente definidos, como son las de ciencias de la salud. Por esta razón, en estas titulaciones, una etapa esencial en el diseño de los planes de estudios basados en competencias es la identificación del perfil o de los correspondientes perfiles profesionales; puesto que serán estos perfiles los que permitirán complementar las competencias comunes a todas o a la gran mayoría de las titulaciones (competencias genéricas o transversales) con aquellas otras que son propias de la titulación (competencias específicas).

A continuación describimos la situación que existe actualmente en España por lo que respecta a la correspondencia entre los objetivos formativos del plan de estudios de la titulación de Medicina y las actividades profesionales para las cuales la obtención del título habilita. Adelantamos ya nuestra opinión en el sentido de que dicha situación dificulta en gran manera el diseño de planes de estudios basados en competencias que sean coherentes.

\section{Salidas profesionales de los titulados en medicina y delimitación de sus funciones}

Si bien en nuestro país las posibles salidas profesionales de los actuales licenciados en medicina han sido y siguen siendo muy diversas [2-5], la gran mayoría de éstos desarrolla exclusivamente actividades asistenciales. Relativamente pocos combinan la actividad asistencial con la investigación y/o la docencia; se dedican en exclusiva a una de estas dos actividades o a ambas de forma simultánea; desarrollan sólo actividades de gestión sanitaria; desempeñan funciones de prevención, de información o de educación sanitarias; realizan funciones de carácter no asistencial en las distintas administraciones públicas, $o$ trabajan en la industria. Por lo que hace a la tarea asistencial, son cada vez más los que la desarrollan dentro del Sistema Nacional de Salud frente a los que la desempeñan vinculados a entidades privadas de asistencia sanitaria o en el ámbito del ejercicio estrictamente privado.

Como señaló la relativamente reciente Ley de Ordenación de las Profesiones Sanitarias (6, Exposición de motivos I), hasta la promulgación de la misma, en España existía una 'situación de práctico vacío normativo' por lo que hace a la regulación del ejercicio profesional de la medicina y del resto de las profesiones sanitarias 'con la sola excepción de la odontología y otras profesiones relacionadas con la salud dental'. Dichas profesiones eran reguladas básicamente a través del régimen de la Organización Colegial correspondiente mediante normas de rango reglamentario. Normas que en el caso de la medicina utilizaban expresiones abstractas como 'la profesión médica,' 'la profesión de médico', 'la práctica de la medicina', y definiciones tan ambiguas como 'se considera como ejercicio profesional la prestación de servicios médicos en sus distintas modalidades' (7, Art. 35.2). La Ley de Ordenación de las Profesiones Sanitarias (LOPS) identificó como 'profesiones sanitarias, tituladas y reguladas, aquellas cuya formación pregraduada o especializada se dirige específica y fundamentalmente a dotar a los interesados de los conocimientos, habilidades y actitudes propios de la atención de salud, y que están organizados en colegios profesionales oficialmente reconocidos por los poderes públicos' (Art. 2.1), Dentro de las mismas, reconoció las profesiones para cuyo ejercicio habilitaban los diversos títulos universitarios a nivel de Diplomado, de Licenciado y de Especialista en Ciencias de la Salud (Art. 2.2), y especificó las funciones propias de cada una de ellas (Arts. 6 y 7). Por lo que respecta a los licenciados en Medicina, estableció que correspondía a éstos 'la indicación y realización de las actividades dirigidas a la promoción y mantenimiento de la salud, a la prevención de las enfermedades y al diagnóstico, tratamiento, terapéutica y rehabilitación de los pacientes, así como al enjuiciamiento y pronóstico de los procesos objeto de atención' (Art. 6.2).

\section{Titulaciones requeridas para el ejercicio profesional}

En todos los países occidentales, el ejercicio profesional de la medicina, como sucede con todas 
las denominadas profesiones tituladas, requiere como requisito legal indispensable la obtención de un título oficial que acredite formalmente la posesión de un núcleo de saberes característico; pero la naturaleza del título acreditativo que se exige varía según el país. En muchos países, las titulaciones universitarias no incorporan como función intrínseca la de habilitar para el ejercicio profesional, sino que ésta depende de otras instituciones ajenas a las universidades, como son las organizaciones profesionales o instituciones de carácter estatal. El grado o diploma universitario es puramente acreditativo de la formación recibida; el acceso a las profesiones y a su ejercicio está condicionado por requisitos extraeducativos, que suelen implicar exámenes profesionales ante el Estado o las correspondientes organizaciones profesionales. Pero ésta no es la situación que se da en España. El ordenamiento jurídico español ha seguido tradicionalmente un criterio general de reglamentación de las profesiones y de vinculación estrecha entre el título universitario y una profesión correspondiente. Tradicionalmente, en nuestro sistema, la obtención del título académico ha bastado y autorizado para la incorporación a la profesión correspondiente, y éste se ha considerado, sin más, como título profesional habilitante a este efecto; si bien, en el caso de las profesiones tituladas y colegiadas, el ejercicio profesional se condiciona a la pertenencia al correspondiente colegio profesional [8].

Por lo que hace al caso de la medicina, la vinculación entre el acceso a la profesión médica y el título universitario se recogió en la legislación española al menos desde la Ley de Instrucción Pública de 1857, conocida como Ley Moyano, y se reiteró en la reforma educativa de 1943. La Ley de Ordenación Universitaria promulgada este año [9] atribuyó a la Universidad, entre otras funciones, la de 'habilitar mediante la investidura de los grados académicos... para el ejercicio de las diversas actividades en los campos de la Administración o de la técnica o para la función docente previo cumplimento de las condiciones legales exigidas para cada caso'. En el desarrollo de esta ley, el Decreto de 7 de julio de 1944, sobre la Ordenación de la Facultad de Medicina [10], estableció que estas facultades tenían 'a su cargo, como parte integrante de la Universidad española, la enseñanza de las ciencias médicas, la consiguiente habilitación de sus alumnos para el ejercicio profesional y el fomento de la inves- tigación científica' (Art. 1), y determinó que 'el título de Licenciado en Medicina será requisito indispensable para el ejercicio profesional de la medicina con carácter general' (Art. 5). Por otra parte, el Decreto, después de afirmar en su exposición de motivos que 'el arduo problema de la especialización había permanecido hasta el momento huérfano de una ordenación sincera, estableció (Arts. 5 y 55) que las facultades de medicina, por medio de los institutos y escuelas profesionales que de ellas dependieran, habilitarían a los licenciados para la expedición por el Ministerio de los títulos de Especialista Médico; título que se exigiría para poder ejercer la medicina con el referido carácter.

La Ley de 20 de julio de 1955, sobre enseñanza, título y ejercicio de las especialidades médicas [11], determinó los requisitos y estudios necesarios para obtener el título de Especialista y exigió la posesión del mismo 'para titularse de modo expreso Médico Especialista y para ocupar cargos con este carácter' (Art. 1); si bien precisó, en la exposición de motivos, que el título de Licenciado en Medicina habilitaba para 'la total práctica de la medicina' sin que los preceptos de la nueva Ley pretendieran 'disminuir su reconocida integridad', y la Orden de 1 de abril de 1958 [12] que desarrolló esta Ley y estableció las normas para la obtención del título de Especialista Médico, insistió en que quien poseyera el título de Licenciado en Medicina podría 'ejercer la Medicina General, la Cirugía General o la medicina en todas o en cualquiera de sus ramas, pero sin poder titularse expresamente especialista en ninguna' (Exposición de motivos y Art. 6).

Como ha señalado Souvirón [13], la Ley General de Educación de 1970 [14] pudo provocar cierto cambio de orientación por lo que hace a los efectos profesionales habilitantes de los títulos universitarios; puesto que al referirse a éstos (Art. 39) no utiliza una expresión de la que pueda derivarse unívocamente una vinculación entre cada título y una correspondiente profesión (p. ej., 'habilitará para el ejercicio de la correspondiente profesión'), sino una fórmula abierta ('habilitará para el ejercicio profesional') de la que podría deducirse que el título no sería configurador de la profesión sino sólo uno de los requisitos legales para el ejercicio profesional, el cual podría exigir otros condicionantes. La Resolución de la Dirección General de Universidades e Investigación, por la que se determinaron las 
directrices de los planes de estudios de las facultades de medicina [15], mantuvo la misma fórmula abierta que la Ley General de Educación, al indicar que el título de Licenciado en Medicina y Cirugía 'capacita para el ejercicio profesional'.

Por lo que se refiere a la formación médica especializada, cabe señalar que la Ley General de Educación determinó que los estudios de especialización de posgrado darían derecho a un certificado acreditativo de los mismos 'con los efectos profesionales que en cada caso se determinen' (Art. 39.4). El incumplimiento de la disposición transitoria 14.2 de la misma Ley, que determinaba que el Gobierno dictaría las normas necesarias para garantizar el uso riguroso tanto con fines profesionales como honoríficos de las titulaciones académicas, dejó en tierra de nadie las previsiones sobre el certificado acreditativo y sus efectos. No obstante, interpretándose que por ello mismo este certificado era también una titulación, la misma pudo servir de base legal para la regulación reglamentaria de títulos profesionales de posgrado (13, p. 83); entre ellos, las especialidades médicas el año 1978.

Se daba la circunstancia de que, por un lado, la Ley General de Educación (Disposición final 4.1) había rebajado a normas de rango reglamentario todas las disposiciones anteriores a la misma; entre ellas, la Ley sobre Especialidades Médicas del año 1955, antes mencionada, y las normas que la habían desarrollado, y de que, por otro lado, a partir de 1971, en las instituciones de la Seguridad Social se había consolidado un sistema formativo paralelo (el de los Médicos Internos Residentes) al término del cual no se otorgaba el título de Médico Especialista previsto en la Ley de 1955 sino un certificado de la formación recibida computable como mérito para ingresar en el sistema de la Seguridad Social (16, p. 31). Por ello, en 1978, los dos sistemas de formación médica especializada se unificaron al promulgarse el Real Decreto 2015/1978, de 15 de julio [17], por el que se reguló la obtención de títulos de especialidades médicas. En dicho R.D. (Art. 1) se mantuvo la previsión de que si bien 'para denominarse de modo expreso Médico Especialista y para ocupar puestos de trabajo en centros e instituciones públicas o privadas con tal denominación' se requería estar en posesión del correspondiente título de especialista, esto no suponía 'perjuicio del libre ejercicio de la profesión que asiste a los licenciados en medicina y cirugía'.
El hecho de que la Constitución Española de 1978 [18], en el Art. 149.1, hablara de títulos académicos y de títulos profesionales fue interpretado por algunos como indicativo de que se reconocían dos categorías de títulos: los títulos académicos, que constituirían el refrendo de un estatus o grado académico, y los títulos profesionales, que serían acreditativos de una cualificación profesional reglamentada. Ál Estado le correspondería establecer los procedimientos oportunos para que los títulos académicos alcanzaran validez profesional (13, p. 251-3). La Ley de Reforma Universitaria (LRU) promulgada el año 1983 [19], que desarrolló la Constitución en este punto, no delimitó la naturaleza académica o profesional de los títulos universitarios, $y$ frente al expreso reconocimiento de efectos profesionales que para los mismos habían hecho las anteriores leyes reguladoras, la LRU no se pronunció a este respecto. Sin embargo, el Real Decreto 1496/1987 [20], dictado en el desarrollo de la LRU, caracterizó a los títulos universitarios oficiales estableciendo que éstos surtirían 'efectos académicos plenos y habilitarían para el ejercicio profesional de acuerdo con la normativa vigente' (Art. 1). Cabe concluir que, de acuerdo con la normativa actual, los títulos universitarios oficiales aún tienen, a la vez, naturaleza académica $\mathrm{y}$ alcance profesional, aunque quede por precisar este alcance (13, p. 252-4 y 306-12).

Por lo que respecta a las especialidades médicas, el Real Decreto 127/1984, de 11 de enero, que reguló la Formación Médica Especializada y la obtención del título de Médico Especialista [21] reprodujo esencialmente la normativa preconstitucional y estableció que dicho título 'sin perjuicio de las facultades que asisten a los licenciados en medicina y cirugía, será obligatorio para utilizar, de modo expreso, la denominación de médico especialista para ejercer la profesión con este carácter y para ocupar un puesto de trabajo en establecimientos o instituciones públicas o privadas con tal denominación' (Art. 1). Pero algunos expertos en derecho constitucional [16] advirtieron que dicho R.D. poseía elementos inconstitucionales. Según tales expertos, el Art. 36 de la Constitución permite que el estado regule el ejercicio de las profesiones tituladas, pero dicha regulación queda sujeta a reserva de ley, lo cual significa que mientras en el derecho preconstitucional la regulación del ejercicio de las profesiones tituladas, entre ellas la medicina, po- 
día llevarse a cabo a través de normas de distinto rango, a partir de la promulgación de la Constitución sólo puede tener lugar mediante una ley formal; es decir, queda excluida del ámbito de la potestad reglamentaria, que sólo podrá complementar la regulación contenida en la ley pero nunca limitar el ejercicio profesional más allá de lo que una ley formal haya dispuesto. En consecuencia, el Art. 1 del R.D. 127/1984, por vulnerar la reserva de ley impuesta por la Constitución, al limitar y condicionar el ejercicio de una profesión, sería nulo de pleno derecho; si bien el resto del articulado, que regula las condiciones para la obtención del título de Médico Especialista en las distintas ramas de la medicina, materia que la Constitución no sujeta a reserva de ley, sería ajustado a derecho [16].

Esta situación de posible inconstitucionalidad quedó subsanada con la promulgación de la Ley 44/2003, de 21 de noviembre, de Ordenación de las Profesiones Sanitarias [6], que recoge (Art. 16.3) como circunstancias que exigen la posesión del título de Especialista Médico aquellas que estaban contempladas en el Art. 1 del R.D. $127 / 1984$

\section{Directivas comunitarias y titulaciones médicas}

Las normas del derecho comunitario, dirigidas a facilitar la libre circulación, establecimiento y prestación de servicios de las personas dentro de la Unión Europea, por una parte, obligan a los estados miembros a incorporar a su derecho interno unas condiciones mínimas que forzosamente ha de reunir la formación médica. Por otra parte, exigen que cada Estado miembro reconozca los títulos expedidos por otro Estado miembro, y les atribuya en su territorio los mismos efectos que a los suyos propios; pero, salvo casos excepcionales, no determinan cuáles han de ser dichos efectos, cuestión que pertenece al ámbito interno de cada Estado [8].

Las primeras directivas referentes a las actividades médicas fueron promulgadas el año 1975; la Directiva 75/362/CEE [22] impuso el mutuo reconocimiento de títulos y la Directiva 75/363/ CEE [23] determinó las características mínimas que debían reunir la formación médica de pregrado y la formación de los médicos especialis- tas. De acuerdo con la Directiva 75/363/CEE, la formación médica de pregrado capacitaba, por una parte, para iniciar los programas de formación médica especializada y, por otra parte, para ejercer las actividades del médico en calidad de generalista sin limitación alguna. Sin embargo, el comité consultivo para la formación de los médicos, creado con fines de asesoramiento [24] después de emitir dos informes sobre las características generales que debía reunir la denominada 'formación médica de base', entendida como la formación teórica y clínica que proporciona los conocimientos que deben ser comunes a todos los médicos con independencia de su orientación futura [25], el año 1979 elaboró un tercer informe en el que concluyó que la formación médica de base no cubría las necesidades requeridas para formarse como médico generalista y recomendó que, con tal fin, se desarrollaran programas de formación específicos [26]. Siguiendo estas recomendaciones, el Consejo de la Comunidad Europea, en 1986, promulgó la Directiva 86/457/CEE [27] y determinó que cada Estado miembro debía establecer una formación específica en medicina general que completase la formación médica de base, de al menos dos años de duración, impartida de acuerdo con su ordenamiento jurídico, o bien en el marco de la formación médica básica o fuera del mismo. Además, el Consejo determinó que a partir de 1995 el ejercicio de las funciones de médico generalista en el marco de un régimen público de seguridad social debería subordinarse a la posesión de la formación específica en medicina general, lo cual supuso una excepción en la normativa comunitaria en el sentido de que se vincularon obligatoriamente unos efectos profesionales a un título académico.

A pesar de haberse promulgado esta normativa sobre la formación específica en medicina general, en la Directiva 2001/19/CEE [28], que alargó de dos a tres años la duración mínima de dicha formación a partir del año 2005, se indicaba que durante el período de formación médica básica deberían haberse adquirido 'conocimientos adecuados en medicina general'. Pero esta expresión se ha sustituido finalmente por la expresión 'conocimientos básicos adecuados de medicina' en la recientemente aprobada Directiva $2005 / 36 / \mathrm{CEE}(29$, p. 59$)$ que agrupa y actuali$\mathrm{za}$ todas las directivas relativas al reconocimiento de las cualificaciones profesionales. 
Para finalizar, indiquemos que si bien hasta ahora no se ha traducido en efectos normativos, el año 1992, en un informe sobre la formación médica de pregrado (30) el comité consultivo recomendó que los programas de la misma deberían diseñarse de manera que quien obtuviera el título 'hubiera adquirido el nivel necesario de conocimientos, técnicas y actitudes que le permitieran participar de forma activa (tal y como legalmente está facultado) en la asistencia y en el tratamiento (investigación para el diagnóstico y terapéutica) de los pacientes, aunque siempre bajo supervisión de sus superiores'. El título o certificado acreditativo expedido al final de los estudios permitiría realizar al menos un año adicional de formación clínica supervisada, que autorizaría para ejercer como profesional independiente.

La normativa comunitaria no supuso ningún problema para España en el momento en que se formalizó su adhesión a la CE. El título español de Licenciado en Medicina y Cirugía fue incorporado de inmediato al listado de títulos de grado para los que se establecía el mutuo reconocimiento, y los títulos correspondientes a las diferentes especialidades médicas existentes en nuestro país fueron incorporados al listado de especialidades mutuamente reconocidas, si bien en este caso fue necesario realizar algunas modificaciones en la legislación española a fin de ajustar la duración de los períodos formativos de ciertas especialidades y las condiciones en que se desarrollaba dicha formación a los requisitos exigidos por las normas comunitarias. Por otra parte, por lo que hace al ejercicio de la medicina general, en cumplimiento de la Directiva 86/457/ CEE que, como se ha comentado, estableció que a partir del año 1995 el ejercicio de las funciones de médico generalista en el marco de un régimen público de seguridad social debería subordinarse a la posesión de una formación específica en medicina general, mediante el Real Decreto 853/1993, de 4 de junio, sobre el Sistema Nacional de Salud [31], se determinó que en España dicha formación específica se acreditaría con el título de Médico Especialista en Medicina Familiar y Comunitaria. Los licenciados antes del primero de enero de 1995 podrían cubrir plazas de Médico de Medicina General en centros o servicios sanitarios integrados en el Sistema Nacional de Salud previa obtención de certificación ministerial que acredita la correspondiente habilitación.

\section{El actual título de Licenciado en Medicina}

El plan de estudios actual de la Licenciatura en Medicina surgió de la reforma de las titulaciones universitarias oficiales realizada en cumplimiento de la Ley de Reforma Universitaria de 1983 [19]. Con tal fin, el Pleno del Consejo de Universidades creó una ponencia para la reforma de las enseñanzas universitarias que estableció 16 grupos de trabajo; el grupo IX fue el encargado de analizar las titulaciones de Medicina, Enfermería y Fisioterapia, y presentar propuestas con vistas a su actualización. Dichas propuestas serían sometidas a consulta pública, valoradas luego por la ponencia y, finalmente, elevadas al Gobierno. El subgrupo del grupo IX encargado de la titulación de Medicina, después de aclarar que el título académico y el título oficial tendrían valor coincidente, a partir de la Directiva 75/363/CEE, de otras propuestas no normativas de la CEE y de los planes de estudios desarrollados en mayor o menor grado como una enseñanza coordinada en algunas facultades de medicina españolas, inició los trabajos que dieron como fruto la propuesta finalmente sometida a consulta pública por el Consejo de Universidades [32,33].

De acuerdo con esta propuesta, la formación médica de pregrado, definida con las mismas palabras con que los documentos comunitarios habían definido la formación médica de base, tendría entre sus objetivos 'preparar médicos bien formados para el ejercicio de sus funciones en el ámbito de la medicina general o de la atención primaria de salud' y 'sentar las bases para el acceso posterior de los alumnos hacia la especialización médica, la investigación científica y la docencia universitaria (...). La orientación de los curricula debería dirigirse hacia la formación de médicos generales competentes, sin perjuicio de que dicha formación pudiera hacerse compatible con la percepción o el conocimiento, en mayor o menor grado, de los fundamentos de la medicina científica'.

El Real Decreto 1417/1990, de 16 de octubre, por el que se establecieron el título de Licenciado en Medicina y las directrices generales de los correspondientes planes de estudios [34,35], suprimió la mayoría de las consideraciones contenidas en la propuesta del grupo IX, pero mantuvo como definición de la formación conducente a la obtención del título correspondiente la definición de formación médica de base contenida 
en las normas comunitarias y fijó como objetivos de la misma los establecidos por la Directiva 75/363/CEE [23].

\section{Reforma del plan de estudios en el marco del EEES}

Con el objetivo de facilitar el diseño de títulos oficiales de grado adaptados al Espacio Europeo de Educación Superior (EEES), la Agencia Nacional de Evaluación de la Calidad y Acreditación (ANECA) inició, el año 2003, el Programa de Convergencia Europea en el que participaron varias redes de universidades y que culminó con la publicación de numerosos 'Libros Blancos' correspondientes a las titulaciones diversas. Las propuestas contenidas en éstos no eran de carácter vinculante, pero, como instrumento de reflexión, fueron presentados al Consejo de Coordinación Universitaria (CCU) y al Ministerio de Educación y Ciencia (MEC) para su información y consideración [36].

El Libro Blanco correspondiente a la titulación de Medicina [37] fue elaborado por la Conferencia Nacional de Decanos de Facultades de Medicina Españolas. En éste se explica que en el proyecto presentado inicialmente a la ANECA se consideraron cuatro perfiles profesionales correspondientes a la titulación: Médico generalista con actividad asistencial, Universidad/ Investigación, Industria Farmacéutica $(\mathrm{I}+\mathrm{D}+\mathrm{I})$ y Gestión Sanitaria. Pero que, como resultado del análisis posterior de dichos perfiles profesionales, la Conferencia de Decanos estimó que la orientación profesional del grado debía ser única 'la correspondiente al denominado "médico de base» en la directiva europea' y que es precisamente a partir de este perfil único y común del que pueden derivarse otros, tras la realización del correspondiente posgrado.

Publicados los decretos que determinaban las nuevas estructuras de las enseñanzas universitarias y regulaban los estudios oficiales de grado y posgrado adaptados al EEES [38,39], el MEC estableció el procedimiento que se debía seguir para la reforma de las titulaciones de grado, y fijó dos etapas: una primera etapa que debía conducir a la propuesta del nuevo catálogo de títulos oficiales y una segunda etapa que finalizaría con la aprobación de las directrices generales propias de cada titulación [40]. Las tareas correspondientes serían desarrolladas por cuatro subcomisiones sectoriales del CCU -entre ellas, la de Ciencias Experimentales y de la Salud [41]- y por una comisión de expertos creada al efecto [42].

Estas subcomisiones realizaron su trabajo entre febrero y junio del año 2005. Analizaron la situación existente, escucharon a diversos interlocutores académicos y sociales, presentaron sus reflexiones y propusieron un mapa preliminar de títulos, a partir del cual el MEC elaboraría las propuestas de directrices generales propias que deberían ser debatidas con todos los sectores implicados en el ámbito de cada titulación. Publicadas dichas directrices, las universidades dispondrían de tres años para elaborar los correspondientes planes de estudios [43].

A principios del año 2006, el MEC remitió al CCU un primer conjunto de propuestas de directrices elaboradas a partir de las aportaciones de las cuatro subcomisiones del Consejo, las conclusiones de redes disciplinares universitarias (Tuning y Libros Blancos), la documentación enviada por diversos colectivos implicados y los programas de estudios afines desarrollados por universidades europeas de prestigio $[44,45]$. En el mes de marzo dio a conocer el calendario de elaboración de las directrices de todas las titulaciones y publicó el procedimiento para el estudio de los títulos de la primera fase $[46,47]$.

La directiva para las enseñanzas de medicina [48] propuso la denominación de 'Médico' para el título y 360 créditos europeos (ECTS) para la formación académica que se debía superar por el estudiante. Indicó como 'efectos académicos del título' que el mismo 'habilitaba para el acceso a los estudios de doctorado y que era necesario para el acceso a la formación sanitaria especializada'. Como 'competencias profesionales que confiere el título’ reprodujo el Art. 6.2 de la Ley de Ordenación de las Profesiones Sanitarias citado al comienzo de este artículo, y precisó que la 'profesión regulada para cuyo acceso habilitaba el título' era la de 'Médico'.

Pero un cambio en la cartera ministerial y la presentación al Parlamento del Proyecto de Ley de modificación de la Ley Orgánica de Universidades [49] determinó un notable cambio de rumbo en el proceso establecido por el MEC para la reordenación de las enseñanzas universitarias, en el sentido de conferir una mayor autonomía a las universidades en el diseño de 
los títulos que imparten [50,51]. Informado el correspondiente borrador por la CRUE, se publicó, con fecha 30 de octubre de 2007, el Real Decreto por el que se estableció la ordenación de las enseñanzas universitarias oficiales [52]. De acuerdo con éste, a partir de unas directrices de carácter muy general, las universidades deberán elaborar las propuestas de los títulos que deseen impartir. El Consejo de Universidades, con la implicación de la ANECA, verificará que los planes de estudios propuestos se adecuen a las directrices y sean coherentes con la denominación de los correspondientes títulos. Y, finalmente, el Gobierno, tras la autorización de la Comunidad Autónoma, establecerá el carácter oficial del título y ordenará su inscripción en el Registro de Universidades, Centros y Títulos (RECUT). Esta inscripción tendrá como efecto la consideración inicial de título acreditado, la cual se mantendrá cuando se obtenga un informe de acreditación positivo transcurridos seis años.

Por otra parte, el Real Decreto determinó (Art. 12.9) que 'cuando se trate de títulos que habiliten para el ejercicio de actividades profesionales reguladas en España, el Gobierno establecerá las condiciones a las que deberán adecuarse los correspondientes planes de estudios, que además deberán ajustarse, en su caso, a la normativa europea aplicable. Estos planes de estudios deberán, en todo caso, diseñarse de forma que permitan obtener las competencias necesarias para ejercer esa profesión. Estableció, además (Disposición adicional 10. a), que los títulos universitarios oficiales 'no podrán inducir a confusión ni coincidir en su denominación y contenidos con los de los especialistas en ciencias de la salud regulados en el capítulo III de la Ley 44/2003, de 21 de noviembre, de ordenación de las profesiones sanitarias'.

De acuerdo con las disposiciones de este Real Decreto, en noviembre de 2007, se dieron a conocer los borradores del Acuerdo del Consejo de Ministros que establecía las condiciones a las que deberían adecuarse los planes de estudios conducentes a la obtención de títulos que habilitasen 'para la profesión regulada de Médico' [53] y de la Orden Ministerial que establecía los requisitos para la verificación de dichos títulos [54], y en diciembre de 2007 y febrero de 2008, respectivamente, se publicaron los textos definitivos de ambos documentos [55,56]. El Acuerdo del Consejo de Ministros determinó que los tí- tulos son 'enseñanzas oficiales de Grado', y que sus planes de estudios deben tener 'una duración de 360 créditos europeos' y deben garantizar 'la adquisición de las competencias necesarias para ejercer la profesión de acuerdo con lo regulado en la normativa aplicable. La Orden Ministerial incorporó, con algunas modificaciones, las competencias específicas que había definido el Libro Blanco sobre la titulación de Medicina [37] y que también había incluido la directiva para las enseñanzas de Medicina del anterior gobierno [48]. Quedó, pues, definitivamente zanjada la discusión de si el título de medicina debía ser sólo de Grado, de Máster o de Grado y Máster.

\section{Consideración final}

Consideramos que la situación a la que se ha llegado mantiene diversas incoherencias que, si no se corrigen, dificultarán el diseño de los nuevos planes de estudios de la titulación en medicina basados en competencias relacionadas con el perfil profesional:

- No puede ignorarse que según doctrina del Tribunal Supremo, 'cualquier médico (sea o no especialista) puede atender cualquier enfermedad de cualquier enfermo (...), ya que el título de especialista sólo es necesario para ejercer la profesión con este carácter.' Quien tenga la titulación en medicina 'puede dedicarse al ejercicio de la medicina, en cualquier ámbito o especialidad, con tal de que no la ejerza con el carácter de especialista' (16, p. 56).

- Es difícil comprender que se mantenga dicha situación cuando se ha llegado a la conclusión de que la formación médica de base proporcionada por la titulación de grado en medicina ni siquiera forma adecuadamente para el desempeño de las funciones propias del médico generalista, para lo cual se requiere una formación complementaria específica. En España, dicha formación específica sólo se exige para ejercer en el marco de los sistemas públicos de Seguridad Social. ¿Es que se considera que los pacientes atendidos en otro marco no requieren médicos con el mismo nivel de competencia?

- Por el hecho de que la Unión Europea no legisle por considerar que ésta es una cuestión 
que han de resolver los estados miembros, ¿pueden aceptar las autoridades ministeriales y académicas españolas que existan en el país dos tipos de medicina general desempeñados por médicos con distinto nivel de competencia?

- Si este no es el caso, ¿es que se considera que la formación correspondiente a la titulación en medicina debe complementar el catálogo competencial propio de la formación básica de médico con las competencias requeridas para el ejercicio adecuado de la medicina general?

- Si esto es así, ¿por qué no se explicita con claridad al definir los planes de estudios de la titulación en medicina y dejan de identificarse los objetivos formativos de dicha titulación con los propios de la formación básica del médico?

- Y si ello no es así, ¿por qué no se elimina mediante ley la habilitación que la simple obtención del título de médico confiere para el ejercicio de la medicina general?

Pensamos no ser los únicos que formulamos estas preguntas, que pueden resultar incómodas a determinados niveles, y queremos creer que el Gobierno, si más no por la vehemente exigencia de las autoridades académicas, acabará respondiendo de forma responsable y rehuyendo toda ambigüedad.

\section{Bibliografía}

1. Perrenoud PH. La universitat entre la transmissió de coneixements i el desenvolupament de competències. Quaderns de Docència Univeristària, n. ${ }^{\circ} 5$. Barcelona: ICE-Universitat de Barcelona; 2005. p. 26-52.

2. Fundación Universidad-Empresa. Monografías Profesionales. Los estudios de Medicina. Madrid; 1979.

3. Profesiones. Conocer y ejercer la medicina. Madrid: Acento Editorial; 1993.

4. Dexeus S. Profesiones con futuro. Médico. Barcelona: Grijalbo; 1994.

5. Roigé J, Sarrias R. Fer de metge a Catalunya. La carrera de medicina avui. Barcelona: Collegi Oficial de Metges de Catalunya; 1998.

6. Ley $44 / 2003$, de 21 de noviembre, de ordenación de las profesiones sanitarias. BOE n. 280 (22/11/2003): 41442-58.
7. Real Decreto 1018/1980, de 19 de mayo, por el que se aprueban los estatutos generales de la Organización Médica Colegial y del Consejo General de Colegios de Médicos. BOE n. ${ }^{\circ} 128$ (28/5/1980): 11559-71.

8. Souvirón JM. La configuración jurídica de las profesiones tituladas en España y en la Comunidad Económica Europea. Madrid: Consejo de Universidades. Secretaría general; 1988.

9. Ley de 29 de julio de 1943 sobre Ordenación de la Universidad Española. Biblioteca Oficial Legislativa, v. 163. Madrid: Instituto Editorial Reus; 1943.

10. Decreto de 7 de julio de 1944 sobre Ordenación de la Facultad de Medicina. BOE n. ${ }^{\circ} 17$ (4/8/1944): 5943-50.

11. Ley de 20 de julio de 1955 sobre Enseñanza, título y ejercicio de las especialidades médicas. BOE n. 202 (21/7/1955): 4440-2.

12. Orden de 1 de abril de 1958 por la que se dictan normas para la obtención del título de especialidades médicas. BOE n. 82 (5/4/1958): 623-4.

13. Souvirón JM. La universidad española. Claves de su definición y régimen jurídico institucional. Universidad de Valladolid. Valladolid: Secretaría de Publicaciones; 1988.

14. Ley General de Educación y Financiamiento de la Reforma Educativa, de 4 de agosto de 1970. BOE n. ${ }^{\circ} 187$ (6/8/1970): 12525-46.

15. Resolución de la Dirección General de Universidades e Investigación por la que se determinan las directrices que han de seguir los planes de estudio de las facultades de medicina. BOE n. ${ }^{\circ} 238$ (4/10/1972): 17687.

16. Herrero de Miñón M, Fernández del Vallado J. Especialización profesión médica. La garantía constitucional de las profesiones tituladas y la especialización médica según la jurisprudencia. Madrid: Civitas; 1997.

17. Real Decreto 2015/1978, de 15 de julio, por el que se regula la obtención de títulos de especialidades médicas. BOE n. 206 (29/8/1978): 2172-4.

18. Constitución Española. BOE n. ${ }^{\circ} 311$ (29/12/1978): 29315-424.

19. Ley Orgánica 11/1983, de 25 de agosto, de Reforma Universitaria. BOE n. 209 (1-/9/1983): 24034-42.

20. Real Decreto 1496/1987, de 6 de noviembre, sobre obtención, expedición y homologación de títulos universitarios. BOE n. 298 (14/12/1987): 36637-9.

21. Real Decreto 127/1984, de 11 de enero, por el que se regula la Formación Médica Especializada y la obtención del título de Médico Especialista. BOE n. ${ }^{\circ} 26$ (31/1/1984): 2524-8.

22. Directiva 75/362/CEE del Consejo, de 16 de junio de 1975 , sobre reconocimiento mutuo de diplomas, certificados y otros títulos de médico. DO n. ${ }^{\circ}$ L 167/14 (30/6/1975): 186-96. 
23. Directiva 75/363/CEE del Consejo, de 16 de junio de 1975, sobre la coordinación de las disposiciones legales, reglamentarias y administrativas referentes a las actividades de los médicos. DO n. ${ }^{\circ}$ L 167/14 (30/6/1975): 197-9.

24. Decisión 75/364/CEE del Consejo, de 16 de junio de 1975 , sobre creación de un Comité consultivo para la formación de médicos. DO n. ${ }^{\circ}$ L 167/17 (16/6/ 1975): 200-1.

25. Commission des Communautés Européennes. Comité Consultatif pour la Formation des Médecins. Rapport sur les tendances générales dans la formation Médicale de base. Juin 1978. Documento III/D/32/1/78-FR.

26. Commission des Communautés Européennes. Comité Consultatif pour la Formation des Médecins. Rapport et avis concernant la formation spécifique du médecine généraliste. Mars 1979. Documento III/D/697/3/79-FR.

27. Directiva 86/457/CEE del Consejo, de 15 de septiembre de 1986, relativa a la formación específica en medicina general. DO n. ${ }^{\circ}$ L 267/26 (19/9/1986): 26-9.

28. Directiva 2001/19/CEE del Parlamento Europeo y del Consejo, de 14 de mayo de 2001, por la que se modifican las Directivas 98/48/CEE y 92/51/CEE del Consejo, relativas al sistema general de reconocimiento de las calificaciones profesionales, y las Directivas 77/452/ CEE, 77/453/CEE, 78/687/CEE, 78/1026/CEE, 78/1027/ CEE, 80/154/CEE, 80/155/CEE, 85/384/CEE, 85/432/ CEE, 85/433/CEE y 93/16/CEE del Consejo, relativas a las profesiones de enfermero responsable de cuidados generales, odontólogo, veterinario, matrona, arquitecto, farmacéutico y médico. DO n. ${ }^{\circ}$ L 206 (31/7/2001): 1-30.

29. Directiva 2005/36/EC del Parlamento Europeo y del Consejo relativa al reconocimiento de cualificaciones profesionales. DO n. ${ }^{\circ}$ L 255 (30/9/2005): 22-142.

30. Comisión de las Comunidades Europeas. Comité Consultivo para la Formación de Médicos. Informe y recomendaciones sobre la formación médica pregraduada. Octubre 1992. Documento III/F/5127/3/92-ES.

31. Real Decreto $853 / 1993$, de 4 de junio, sobre el ejercicio de las funciones de médico de Medicina General en el Sistema Nacional de Salud. BOE n. 262 (1/7/1993): 20049-51.

32. Oliva H. La reforma de los planes de estudio en las facultades de medicina españolas. Rev Clin Esp 1987; 181: 445-52.

33. Consejo de Universidades. Reforma de las Enseñanzas Universitarias. Título de Licenciado en Medicina y Cirugía. Madrid: Consejo de Universidades. Secretaría general; 1988. p. 22-43.

34. Real Decreto 1417/1990, de 26 de octubre, por el que se establece el título universitario oficial de Licenciado en Medicina y las directrices generales propias de los planes de estudios conducentes a la obtención de aquél. BOE n. 278 (20/11/1990): 34343-5.

35. Real Decreto 371/2001, de 6 de abril, por el que se modifican parcialmente diversos reales decretos por los que se establecen títulos universitarios oficiales y las directrices generales propias de los planes de estudios conducentes a la obtención de aquellos. BOE n. ${ }^{\circ} 101$ (27/4/2001): 15343-51.

36. ANECA. Programas/Programa de Convergencia Europea/Libros blancos. URL: http://www.aneca.es/modal_ eval/conver_docs_titulos.html.

37. ANECA. Libro blanco del título de grado en Medicina. URL: http://www.aneca.es/modal_eval/docs/libroblanco_medicina_def.pdf.

38. Real Decreto 55/2005, de 21 de enero, por el que se establece la estructura de las enseñanzas universitarias y se regulan los estudios universitarios oficiales de grado. BOE n. ${ }^{\circ} 21$ (25/1/2005): 2842-6.

39. Real Decreto 56/2005, de 21 de enero, por el que se regulan los estudios oficiales de posgrado. BOE n. ${ }^{\circ} 21$ (25/1/2005): 2846-51.

40. MEC. Procedimiento a seguir tras la aprobación del Real Decreto por el que se establece la estructura de las enseñanzas universitarias y se regulan los estudios universitarios de grado. URL: http://www.upf.edu/web/docencia/docencia.htm?opcio $=4$.

41. Proceso de elaboración del catálogo de titulaciones. URL: http://www.crue.org/catalogo_titulaciones.htm.

42. MEC. La comisión de expertos que estudiará las propuestas de mapa de titulaciones celebrará mañana su reunión constitutiva. Nota de prensa (19/7/05). URL: http://www.mec.es/mecd/gabipren/index.html.

43. Comisión Externa (21/9/05). Acuerdo sobre el proceso de renovación de las enseñanzas universitarias para su adaptación al Espacio Europeo de Educación Superior: principios, criterios y calendario. URL: http://www.upf. edu/web/docencia/docencia.htm?opcio=4.

44. MEC. El MEC remite al Consejo de Coordinación Universitaria las primeras directrices de títulos adaptados al Espacio Europeo. Nota de prensa (20/1/06). URL: http:// www.mec.es/mecd/gabipren/index.html.

45. Presentación de la propuesta de directrices generales propias de los estudios de grado. URL: http://www.upf. edu/web/docencia/docencia.htm?opcio=4.

46. Propuesta para la renovación del actual catálogo de títulos: relación y calendario. URL: http://www.upf.edu/ web/docencia/docencia.htm?opcio $=4$.

47. Procedimiento para el estudio de las propuestas de títulos de la primera fase. URL: http://www.upf.edu/web/ docencia/docencia.htm?opcio $=4$.

48. Ficha técnica de propuesta de título universitario según RD 55/2005, de 21 de enero. Enseñanzas de Medicina. URL: http://www.crue.org/boletines/ boletin_n5/boletín\%20N`5/adjuntos/ficha\%20medicina.pdf.

49. MEC. Mercedes Cabrera, nueva ministra de Educación y Ciencia. Nota de prensa. URL: http://www.mec.es/mecd/ gabipren/index.html [07.04.2006]. 
50. MEC. El Gobierno aprobará directrices que permitirán a las universidades impartir los nuevos títulos de grado de 4 años. Nota de prensa. URL: http//www.mec.es/mecd/ gabipren/index.html [05.07.2006].

51. MEC. La organización de las enseñanzas universitarias en España. URL: http//www.mec.es/educa/jsp/plantilla. jsp? area $=$ ccuniv\&id $=920$ [26.09.2006].

52. Real Decreto 1393/2007, de 29 de octubre, por el que se establece la ordenación de las enseñanzas universitarias oficiales. BOE núm. 260 (30/10/2007): 44037-48.

53. Borrador de Acuerdo de Consejo de Ministros por el que se establecen las condiciones a las que deberán adecuarse los planes de estudios conducentes a la obtención de títulos que habiliten para el ejercicio de la profesión regulada de Médico (21/11/2007). URL: http://webs.uvigo. es/victce/images/documentos/normativas/medicina.doc [15/09/2008]
54. Borrador de Orden Ministerial por la que se establecen los requisitos para la verificación de los títulos que habiliten para el ejercicio de la profesión regulada de Médico (21/11/2007). URL: http://webs.uvigo.es/victce/images/documentos/normativas/ficha_medicina2.doc [15/09/2008].

55. Resolución, de 17 de diciembre de 2007, de la Secretaria de Estado de Universidades e Investigación, por la que se publica el Acuerdo del Consejo de Ministros de 14 de diciembre de 2007, por el que se establecen las condiciones a las que deberán adecuarse los planes de estudios conducentes a la obtención de títulos que habiliten para el ejercicio de la profesión regulada de Médico. BOE núm. 305 (21/11/2007): 52849-50.

56. Orden ECI/332/2008, de 13 de febrero, por la que se establecen los requisitos para la verificación de los títulos universitarios que habiliten para el ejercicio de la profesión regulada de Médico. BOE núm. 40 (15/2/2008): 8351-5. 